\title{
A new link between pesticides and Parkinson's disease
}

\author{
Benoit I. Giasson and Virginia M.-Y. Lee \\ Environmental factors are thought to be an important cause of Parkinson's disease. A new
study shows that rats chronically treated with the mitochondrial inhibitor rotenone, a
common pesticide, develop neuropathological and behavioral symptoms of Parkinsonism.
}

Parkinson's disease (PD) is one of the most common neurodegenerative diseases, affecting almost $1 \%$ of the population over 65 . Although the symptoms and neuropathology of PD have been well characterized, the underlying mechanisms and causes of the disease are still not clear. In this issue, Betarbet et al. ${ }^{1}$ report that chronic administration of rotenone, a widely used pesticide, can induce the major features of PD in rats. This remarkable observation not only provides a new animal model for the study of PD, but will also reinvigorate interest in the possible role of exposure to pesticides and other toxins as a cause of human neurodegenerative disease.

$\mathrm{PD}$ is characterized clinically by rigidity, tremor and bradykinesia, which result from the progressive death of dopaminergic neurons in the substantia nigra. The defining feature of idiopathic PD is the accumulation of Lewy bodies in the substantia nigra and other brainstem nuclei ${ }^{2}$. Classical Lewy bodies are small $(5-25 \mu \mathrm{m})$ spherical inclusions, consisting of a dense granular core surrounded by a halo of radiating filaments, and it is suspected that these deposits are involved in triggering the death of dopaminergic neurons.

The etiology of PD is diverse and complex. Some cases can be attributed to genetic factors, and several mutations have recently been described that lead to familial $\mathrm{PD}^{3}$. Two of these mutations, in the genes encoding parkin and ubiquitin carboxy-terminal hydrolase-L1 (UCH-L1), lead to diseases that are somewhat distinct, both clinically and pathologically, from idiopathic cases, and how they relate to classical PD remains

The authors are in the Center for Neurodegenerative Disease Research, Department of Pathology and Laboratory Medicine, University of Pennsylvania, Philadelphia, Pennsylvania 19104, USA. e-mail:vmylee@mail.med.upenn.edu or giassonb@mail.med.upenn.edu unclear. The third class of mutations, in contrast, has been more informative. These affect the gene for $\alpha$-synuclein, a synaptic protein of unknown function that subsequently proved to be a major component of the filaments associated with Lewy bodies. In addition, $\alpha$-synuclein is the major constituent of several other disease-specific lesions, including cortical Lewy bodies, swollen neurites (known as Lewy neurites) and glial cell inclusions ${ }^{4}$. The term 'synucleinopathy' has been coined to designate this broad class of neurodegenerative disorders, and the genetic link between $\alpha$-synuclein and familial PD suggests that Lewy body formation may be an important trigger in the disease process. Further evidence for this idea comes from experiments in which $\alpha$-synuclein is overexpressed in flies or mice. Mutant flies carrying the human gene undergo adult-onset loss of dopaminergic neurons, accompanied by formation of filamentous $\alpha$-synuclein inclusions as well as locomotor dysfunction $^{5}$. In mice, overexpression of human $\alpha$-synuclein results in impaired motor function, neuronal degeneration and perikaryal, neuritic and (unexpectedly) nuclear $\alpha$-synuclein aggregates. These deposits, however, are not filamentous ${ }^{6,7}$, suggesting that the mechanisms leading to polymerization of $\alpha$-synuclein fibrils are more complex than mere protein accumulation.

Genetic mutations can provide important clues to disease mechanism, but most PD cases are sporadic rather than famil-

Fig. 1. Possible mechanisms of selective cell death in Parkinson's and related diseases. Mutations in $\alpha$-synuclein may enhance its propensity to polymerize, resulting in the formation of inclusions that may comprise neuronal viability. Unidentified genetic mutations or polymorphisms may contribute directly to cell death or modulate the propensity of $\alpha$-synuclein to form inclusions, and they may also affect vulnerability to oxidative damage. Known mutations in genes such as U CH-L1 and parkin lead to the death of dopaminergic neurons, but it is unclear whether $\alpha$-synuclein pathology is involved. Environmental factors may also induce cell death, either directly or by initiating pathological cascades involving oxidative damage and $\alpha$-synuclein aggregation.

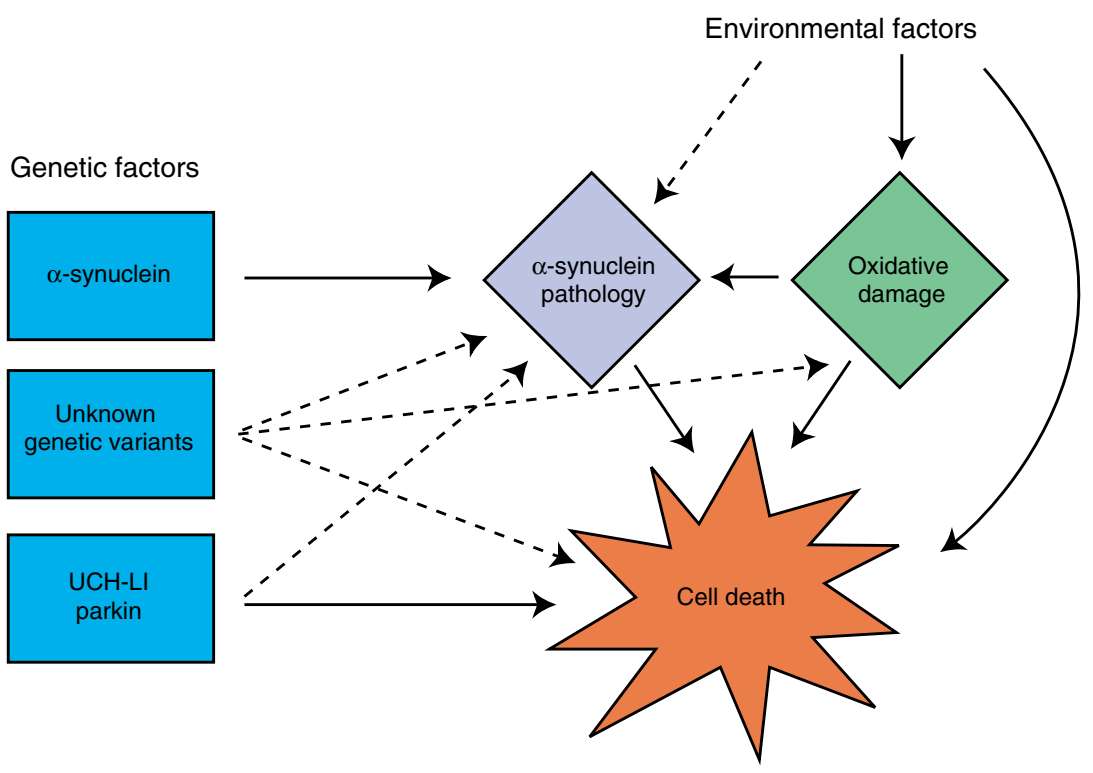


ial, and environmental factors have long been suspected to contribute to the disease. One possibility is that an infectious agent may be responsible-Parkinsonism can follow von Economo's encephalitis, a disease that reached pandemic levels in the early twentieth century-but no infectious agent has ever been identified ${ }^{8}$. An alternative possibility is that a toxin may be involved. Interest in this idea was driven by the appearance, in the early 1980s, of PD-like symptoms, including damage to the nigrostriatal dopaminergic system, in heroine addicts who had been exposed to 1-methyl-4-phenyl-1,2,3,6-tetrahydropyridine (MPTP), a substance inadvertently produced during the attempt to synthesize a meperidine analog as a 'designer drug' ${ }^{9,10}$. MPTP induces acute and irreversible PD-like symptoms in monkeys as well as humans, but although its effects resemble those of idiopathic $\mathrm{PD}$ in many respects, providing a useful animal model, it does not induce Lewy body formation, either in humans or in animals ${ }^{10,11}$.

MPTP is toxic because it is converted into 1-methyl-4-phenylpyridinium ion (MPP+), which is selectively imported into dopaminergic neurons by the plasma membrane dopamine transporter. MPP+ accumulates in mitochondria, where it inhibits complex I (also known as the NADH dehydrogenase complex), one of the enzymes involved in oxidative phosphorylation $^{12}$. This raises the possibility that other inhibitors of complex I might also induce PD-like symptoms. Betarbet et al. ${ }^{1}$ have now tested this hypothesis using rotenone, a plant-derived inhibitor of complex I that is widely used both as a household insecticide and as a tool for eradicating fish (as part of fishery management programs).

The authors show that chronic i.v. administration of rotenone in rats produces effects that closely resemble human PD. The treated rats showed several cardinal behavioral features of the disease, including bradykinesia, postural instability, unsteady gait and some evidence of tremor. Histological examination indicated that rotenone treatment leads to progressive degeneration of the nigrostriatal dopaminergic system. Importantly, the surviving nigral neurons developed intracytoplasmic $\alpha$-synuclein-rich inclusions that appeared similar to Lewy bodies by light and electron microscopy. Although some of these inclusions resembled 'pale bodies', presumptive precursors of Lewy bodies, others showed the characteristic features of authentic human Lewy bodies, including a distinct core surrounded by a fibrillar halo.

Unlike $\mathrm{MPP}+$, which targets dopaminergic neurons because of its affinity for the dopamine transporter, rotenone can cross cell membranes and is therefore likely to affect all cells. Consistent with this, the authors confirmed that their treatment protocol led to complex I inhibition throughout the brain. However, they observed PD-like symptoms even at low concentrations, for which the degree of complex I inhibition is insufficient to significantly impair oxidative phosphorylation in the brain. This argues against ATP depletion as a mechanism for the loss of dopaminergic neurons. An alternative possibility, consistent with earlier suggestions ${ }^{13}$, is that free radicals, such as superoxide anions, which are known to result from mitochondrial inhibition, may be responsible for the damage. Oxidative damage has long been implicated in neurodegenerative diseases, and it may be of particular importance in synucleinopathies, given the extensive and widespread oxidative modification of $\alpha$-synuclein in the deposits characteristic of these diseases ${ }^{14}$. Moreover, oxidative damage is at least partly responsible for the toxic effects of MPTP.

The question remains as to why rotenone, which inhibits complex I throughout the brain, should preferentially target dopaminergic neurons. The likely answer is that these neurons are particularly sensitive to oxidative stress because of the permanently elevated level of free radicals generated by dopamine metabolism and auto-oxidation ${ }^{15}$. This may not be the whole story, however, because the authors observed differences in rotenone vulnerability between different populations of dopaminergic neurons within the substantia nigra, and between dopaminergic fiber projections and cell bodies ${ }^{1}$. Clearly, there may be other factors at work, which remain to be clarified by further study.

Figure 1 summarizes some of the complex mechanisms that may be involved in PD and related diseases. The importance of $\alpha$-synuclein Lewy body pathology is not fully understood, but it is likely that the accumulation of $\alpha$-synuclein filaments, possibly as a consequence of oxidative damage, contributes to impairment of cellular function, eventually resulting in cell death. Unraveling the interplay of genetic and environmental factors will be crucial in dictating the future direction of research and in guid- ing the development of novel therapies for synucleinopathies. The results of Betarbet et al. ${ }^{1}$ should provide a valuable new model for assaying the efficacy of such treatments.

The new study also has important epidemiological implications. Rotenone is a naturally occurring substance that is eventually degraded in the environment, and as such it is considered to be benign compared to many other pesticides. The results of Betarbet et al. are likely to raise new questions about its safety, although whether rotenone exposure contributes to the incidence of $P D$ remains to be determined. Nevertheless, the effects of chronic rotenone administration observed here may be representative of the possible effects of exposure to low amounts of other environmental toxins, yet to be identified. Although genetic factors certainly contribute to vulnerability, the most important risk factor for PD is age, consistent with the idea that chronic exposure to low levels of noxious substances over time may drive a molecular chain of events that eventually leads to PD. Chronic effects may also be compounded by episodes of acute neuronal death, perhaps as a result of transient toxin exposure. The new study will revitalize the search for environmental toxins, including other pesticides, that may contribute to the etiology of this disease.

1. Betarbet, R. et al. Nat. Neurosci. 3, 1301-1306 (2000).

2. Forno, F. S. J. Neuropathol. Exp. Neurol. 55, 259-272 (1996).

3. Dunnett, S. B. \& Björklund, A. Nature 399, Supp, A32-A39 (2000).

4. Duda, J. E., Lee, V. M.-Y. \& Trojanowski, J. Q. J. Neurosci. Res. 61, 121-127 (2000).

5. Feany, M. B. \& Bender, W. W. Nature 404, 394-398 (2000).

6. Masliah, E. et al. Science 287, 1265-1269 (2000).

7. Van der Putten, H. et al. J. Neurosci. 20, 6021-6029 (2000).

8. Casals, J., Elizan, T. S. \& Yahr, M. D. J. Neural Transm. 105, 645-676 (1998).

9. Langston, J. W., Ballard, P. A., Tetrud, J. W. \& Irwin, I. Science 219, 979-980 (1983).

10. Langston, J. W. et al. Ann. Neurol. 46, 598-605 (1999).

11. Forno, L. S., DeLanney, L. E., Irwin, I. \& Langston, J. W. Adv. Neurol. 69, 217-228 (1996).

12. Przedborski, S. \& Lewis-Jackson, V. Mov. Dis. 13, 35-38 (1998).

13. Beal, M. F. Curr. Opin. Neurobiol. 6, 661-666 (1996).

14. Giasson, B. I. et al. Science 290, 985-988 (2000).

15. Olanow, C. W. Neurology 40 Suppl 3, 32-39 (2000). 